\title{
Spinal magnetic resonance imaging with reduced specific absorption rate in patients harbouring a spinal cord stimulation device - A single-centre prospective study analysing safety, tolerability and image quality
}

\author{
Mutter, U M ; Bellut, D ; Porchet, F ; Schuknecht, B
}

\begin{abstract}
BACKGROUND:Spinal cord stimulation (SCS) is an accepted treatment in patients with failed back surgery (FBS), complex regional pain syndrome (CRPS) and persistent radicular pain following surgery. In order to avoid patient hazards or device malfunction manufacturers advise to abstain from magnetic resonance imaging (MRI) in patients with implanted electrodes or pulse generators. METHODS:In a prospective study, 13 patients harbouring an implanted Medtronic Spinal Cord Stimulation (SCS) device underwent MRI $(1.5 \mathrm{~T})$ of the lumbar $(\mathrm{n}=13)$, the cervical $(\mathrm{n}=2)$ or the thoracic spine $(\mathrm{n}=1)$ following the development of new spinal symptoms. An adapted MRI protocol was used limiting the transmitted energy and specific absorption rate. Tolerability and safety were assessed by means of a standardized patient evaluation form documenting pain on a visual analogue scale (0-10), neurologic deficit, and discomfort during the scan. In addition, overall satisfaction with the examination procedure was rated on a Likert scale (1-5). Image quality was rated independently and blinded to the presence of a SCS device by the radiologist and the surgeon as equivalent, superior or inferior compared to the standard spine MRI examination. RESULTS:None of the 13 patients investigated by the modified spinal MRI protocol experienced new neurological deficits, worsening of symptoms or a defect/malfunction of the implant device. Three patients $(23.1 \%)$ reported transient warm sensation in the location of the electrode and in one case intermittent slight tingling in the lower extremities. Overall satisfaction with the examination was $1.13 \pm 0.34$ according to Likert scale (1-5). The image quality was rated - not statistically significant - slightly inferior to standard lumbar spine imaging $(0.82 \pm 0.54)$ with a kappa value of 0.68 between the two investigators. MRI examinations detected relevant and new lesions in 9 (69.2 $\%)$ patients which affected treatment in $8(61.5 \%)$ individuals. CONCLUSION:Using a protocol with a reduced specific energy absorption rate, spinal MRI examinations in patients with SCS can be considered safe. The current view that neurostimulators are a general contraindication to MR examinations has to be reconsidered in patients with new or progressive spinal symptoms.
\end{abstract}

DOI: https://doi.org/10.1007/s00701-013-1885-8

Posted at the Zurich Open Repository and Archive, University of Zurich

ZORA URL: https://doi.org/10.5167/uzh-87052

Journal Article

Accepted Version

Originally published at:

Mutter, U M; Bellut, D; Porchet, F; Schuknecht, B (2013). Spinal magnetic resonance imaging with reduced specific absorption rate in patients harbouring a spinal cord stimulation device - A single-centre 
prospective study analysing safety, tolerability and image quality. Acta Neurochirurgica, 155(12):23272332.

DOI: https://doi.org/10.1007/s00701-013-1885-8 
Spinal magnetic resonance imaging with reduced specific absorption rate in patients harbouring a spinal cord stimulation device -

A single centre prospective study analysing safety, tolerability and image quality

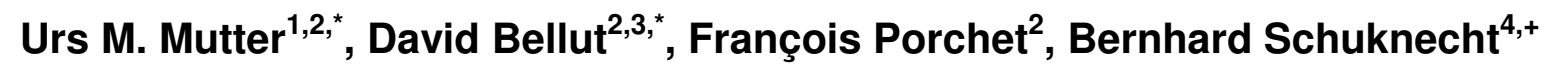

${ }^{1}$ Neuro- und Wirbelsäulenzentrum, Hirslanden Klinik St. Anna, Lucerne

${ }^{2}$ Spine Center, Schulthess Clinic, Zurich, Switzerland

${ }^{3}$ Department of Neurosurgery, University Hospital of Zurich, Switzerland

${ }^{4} \mathrm{MRI}$ Institute, Zurich Switzerland

No disclosure. No conflict of interest.

*Authors contributed equally

${ }^{+}$Corresponding author: Dr. med. D. Bellut

Department of Neurosurgery, University Hospital of Zurich dbellut@gmail.com 


\section{Abstract}

\subsection{Background:}

Spinal Cord Stimulation (SCS) is an accepted treatment in patients with Failed Back Surgery (FBS), Complex Regional Pain Syndrome (CRPS) and persistent radicular pain following surgery. In order to avoid patient hazards or device malfunction manufacturers advise to abstain from magnetic resonance imaging (MRI) in patients with implanted electrodes or pulse generators.

\subsection{Methods:}

In a prospective study 13 patients harbouring an implanted Medtronic Spinal Cord Stimulation (SCS) device underwent MRI (1.5T) of the lumbar $(n=13)$, the cervical $(n=2)$ or the thoracic spine $(n=1)$ following the development of new spinal symptoms. An adapted MRI protocol was used limiting the transmitted energy and specific absorption rate.

Tolerability and safety were assessed by means of a standardized patient evaluation form documenting pain on a visual analogue scale (0-10), neurologic deficit and discomfort during the scan. In addition, overall satisfaction with the examination procedure was rated on a Likert scale (1-5). Image quality was rated independently and blinded to the presence of a SCS device by the radiologist and the surgeon as equivalent, superior or inferior compared to the standard spine MRI examination.

\subsection{Results:}

None of the 13 patients investigated by the modified spinal MRI protocol experienced new neurological deficits, worsening of symptoms or a defect/malfunction of the implant device. Three patients (23.1\%) reported transient warm sensation in the location of the electrode and in one case intermittent slight tingling in the lower extremities. Overall satisfaction with the examination was $1.13 \pm 0.34$ according to Likert scale (1-5). The image quality was rated - not statistically significant - slightly inferior to standard lumbar spine imaging $(0.82 \pm 0.54)$ with a kappa value of 0.68 between the two investigators. MRI examinations detected relevant and new lesions in $9(69.2 \%)$ patients which affected treatment in $8(61.5 \%)$ individuals.

\subsection{Conclusion:}

Using a protocol with a reduced specific energy absorption rate, spinal MRI examinations in patients with SCS can be considered safe. The current view that neurostimulators are a general contraindication to MR examinations has to be reconsidered in patients with new or progressive spinal symptoms. 


\subsection{Keywords:}

Spine, Spinal Cord Stimulation, SCS, MRI, magnetic resonance imaging

\section{Introduction:}

Spinal Cord Stimulation was introduced as a new treatment for chronic pain of spinal origin in 1967 when Shealy implanted a dorsal column stimulator in a patient with pain due to cancer [20]. The principle of the technique is to inhibit afferent nerve fibre activation due to the gate control therapy [15]. SCS has been established as an accepted treatment in patients with Failed Back Surgery (FBS) [17, 22], Complex Regional Pain Syndrome (CRPS) [2, 10] , persistent radicular pain following surgery and peripheral ischemic limb pain $[1,3,4,8,11]$ with a considerable number of patients having undergone implantation of Spinal Cord Stimulators (SCS) [7].

Manufacturer of SCS and the health administration advise not to perform MRI in patients with implanted electrodes or pulse generators implanted [13, 14, 24]. In case of new symptoms or neurologic deficits alternative diagnostic techniques such as CT, myelography or CT myelography harbour the disadvantage of being more invasive and less accurate of particularly following previous spine surgeries.

Only few studies investigated the use of spinal MRI in patients with SCS or intrathecal pump system $[5,6,9,12,16,23]$ demonstrating feasibility and safety but higher patient numbers or effects of implanted SCS on image quality has not been studied. Hazards may arise from the static magnetic field that may exert forces on the implanted devices. The pulsed gradient magnetic fields may cause thermal tissue damage and malfunction of the device.

Despite recent observations stating the safety under particular precautions safety warnings of the manufacturers and the national health administrations [13, 14, 24] refrain most physicians from referring patients with implanted SCS to MRi examinations and radiology departments - considering the SCS an absolute contraindication do not accept these patients for MRI investigations.

Based on previous observations of safety of the spinal MRI (13-15) the objective of the present study was to evaluate the safety and tolerability of a specifically adapted MRI protocol in patients harbouring an implanted SCS device in the clinical setting of new symptoms. A further objective was to assess the clinical consequences, and the image quality of these MRI studies 


\section{Methods:}

\subsection{Study design:}

Following the development of new spinal symptoms 13 patients with implanted Medtronic SCS devices (all IPG placed at the buttocks) were prospectively investigated by MRI of the lumbar $(n=13)$, cervical $(n=2)$ or the thoracic spine $(n=1)$ using a 1.5T Siemens Magnetom Aera MRI scanner Institutional approval of this study for the study and consent had been obtained in every patient. In case of neurological deficits or non-conclusive previous CT studies MRI was indicated and performed using a specific adapted MR protocol. The Internal Pulse generator (IPG) of the SCS device had been deactivated before the examination. Following the MRI the patients completed questionnaires for evaluation of the procedure and the SCS was activated again by a check of the program functions by the manufacturer's technician. A clinical control was conducted by the same spine surgeon. The image quality was compared to the routine spinal MRI protocol by a Neuroradiologist and a Surgeon.

\subsection{Imaging studies:}

Patients were examined in a single radiological centre (Neuroradiology Section, MRI Institute Zurich Switzerland) on a 1.5T Magnetom Aera MR scanner Siemens Healthcare, Erlangen, Germany

The adapted protocol limited the specific absorption rate (SAR) of transmitted energy to $14-37 \%$ in the exposed body region in comparison to $50-79 \%$ applied by the normal spine MR protocol. The specific absorption rate is the energy being absorbed by the body. It is measured in Watts per kilogram and used for various devices emitting radiofrequencies. For example, for mobile phones there is a maximum SAR of $1.6 \mathrm{~W} / \mathrm{kg}$ in the USA and $2.0 \mathrm{~W} / \mathrm{kg}$ in the European Union. The protocol comprised the sequences and parameters shown in table 5. No fat saturation pulse was applied; the positioning mode was put on "reference" instead of "isocentre".

\subsection{Evaluation of tolerability of MRI examination}


Tolerability and safety were assessed immediately after the examination by means of a questionnaire and a standardized patient evaluation form documenting pain on a visual analogue scale (0-10), neurologic deficit and discomfort during and right after the MR examination. In addition, overall satisfaction with the examination procedure was rated on a Likert scale (1-5). Image quality was rated independently by the radiologist and the surgeon as equivalent, superior or inferior compared to a routine spine MR examination of standard quality.

\section{Results:}

\subsection{General patients' data: (Table 1)}

The thirteen patients underwent a mean 2.67 (range: 0-6) operations of the lumbar or cervical spine or the upper or lower extremity before SCS implantation due to persisting neuropathic pain. These included discectomy for herniated disc, lumbar interlaminar decompression spinal canal stenosis and stabilization and fusion for degenerative disc disease and severe spondylarthritis. 11 patients underwent SCS implantation at the lower thoracic spine for symptoms of lumbar spine origin, one patient for treatment of neuropathic pain due to repeat foot surgery and one patient after various carpal tunnel surgeries. There were 9 (69.2\%) female and $4(30.7 \%)$ male patients with a mean age of $56.46 \pm 12.79$ years at time of the MRI examination for the entire series.

\subsection{Tolerability of MRI examination (Table 2):}

The examination was well tolerated by every of the 13 patients. Three patients (23.1\%) reported transient warm sensation in the location of the electrode and in one case intermittent slight tingling in the lower extremities was reported.

The overall level of discomfort ( $0-10 ; 0$ - lowest, 10 - highest) was $2.00 \pm 2.13$ on a visual analogue scale and the overall satisfaction with the scan (; 1- highest, 5 lowest) was $1.13 \pm 0.34$.

None of the patients developed newly symptoms and there was no stimulator dysfunction after the MRI.

\subsection{MRI findings and therapeutic consequences (Table 3):}

There were 9 out of 13 patients with new imaging findings. Those included 4 cases (23.5\%) with high grade spinal canal stenosis, 3 cases (17.6\%) with disc herniation 
and 5 cases $(29.4 \%)$ of severe single segment degeneration with disc protrusion, facet joint arthrosis and osteochondrosis. The imaging studies provided an indication for further invasive therapy in 8 patients. Three patients $(23.1 \%)$ underwent epidural or facet joint infiltration, two patients (15.4\%) underwent lumbar decompression, two patients (15.4\%) underwent transpedicular stabilization and fusion (T-LIF) and one patient $(7.7 \%)$ underwent microsurgical discectomy.

\subsection{Imaging studies and image quality:}

Image quality was rated by the Neuroradiologist and the Surgeon. Images were rated in comparison to standard lumbar spine MR study of normal quality. The different sequences of all imaging studies of all patients were rated independently ( 0 - quality inferior-, 1 - quality equal-, 2 - quality superior to standard imaging study. We found a - statistically not significant - slightly lower imaging quality compared to the standard imaging study. The mean values were $0.80 \pm 0.60$ for the T2-sagittal sequences, $0.96 \pm 0.52$ for the T2-coronal sequences, $0.77 \pm 0.56$ for the T2-axial sequences and $0.75 \pm 0.43$ for the T1-sagittal sequences. Overall mean value for image quality was $0.82 \pm 0.54$. The inter-rater agreement calculated by Fleiss method showed the following kappa-values: 0.73 for the T2-saggital sequences, 0.62 for the T2-coronal sequences, 0.73 for the T2-axial sequences and 0.64 for the T1-sagital sequences. Overall inter-rater agreement showed a kappa of 0.68 .

\section{Discussion:}

The need for high resolution imaging like MRI is rising with an increasing number of patients harbouring implanted neurostimulation devices. Many of these patients are contained within a long term therapy program which facilitates close surveillance in case of a new clinical event necessitating medical or surgical treatment. Until recently, MRI studies were considered contraindicated or at least not recommended by manufacturers and healthcare administrations [24, 25]. The reasons are the use of ferromagnetic materials that might interact with the magnetic field and displacement or uncontrolled heating of both the IPG or the electrode that may occur.

Manufacturer taking efforts in developing devices meeting MRI safety recommendations and recently percutaneous leads are on their way into clinical use. Nevertheless surgical leads cleared for use in an MRI are still unavailable. 
Several investigations on the effect of MRI on implant carriers with Deep brain stimulation (DBS) or cardiac pacemakers are available. Information on the effect of MR on implanted spinal cord stimulator devices is rarely available. A 2002 in vitro study by Kainz et al revealed a temperature increase of $2.1^{\circ} \mathrm{C}$ (whole body SAR 2 $\mathrm{W} / \mathrm{kg}$ ) at the tip of the leads using a 1.5 and a 3 Tesla scanner on an ITREL III and standard electrodes by Medtronic [9]. Usually a range of $45^{\circ} \mathrm{C}$ is described to be at risk for irreversible damages on tissue of the central nervous system (CNS). Due to these minor changes the procedure was considered not to be harmful to the patient. Rezai et al. [18] in contrast found a temperature increase of $7.1^{\circ} \mathrm{C}$ (whole body SAR $3.9 \mathrm{~W} / \mathrm{kg}$ ) and up to $25^{\circ} \mathrm{C}$ at the IPG case.

In our series only three patients reported a warming sensation at the location of the IPG (VAS 3/10 and $5 / 10$ ) but no clinical events were reported.

The devices under investigation are all from one manufacturer (Medtronic). This limitation is due to the pre-, peri- and post interventional support needed. The products are equipped with an electromagnetic interference circuit protection filtering high frequency energy - a so called filtered feed through. The amount of ferrous material is reduced to the parts like antenna or recharge coil to minimize the magnetic pulling. This reduces the risk of circuit breakage and dislocation. Finally the recent generation of neuromodulation devices does not carry magnetic reed switches that can be damaged during MRI procedure. While all the neurostimulators on the market look similar the safety labelling of one company does not apply for the other and other devices are equipped with other safety features. All of the systems still have to manage the RF lead heating risk due to the unavoidable unintentional antenna that the leads and extensions create.

Regarding reports of unintentional activation of the system [19, 21] no activation was found in our series. One patient reported twitches during the scan but no noticeable problems could be detected in the system check. Anecdotic reports of high powered stimulation of non deactivated stimulators are available. The recommend is that the IPG should be deactivated during the procedure.

Image Quality was comparable and not statistically different from the quality derived from an optimized standard spine imaging protocol. The quality of T2 weighted sequences which provide the highest diagnostic yield was closer to the quality standard protocol than the sagittal T1 weighted sequence. Particular sequences like 
fat suppressed contrast enhanced T1 weighted series that apply a high specific absorption rate have to be avoided. However, these are not a component of the standard spine protocol either

Only few papers are available discussing the use of MRI in patient with implanted SCS like the study by De Andres et al [5]. We followed his recommendations regarding reduction of specific absorption rate and furthermore aimed to quantify patient safety and satisfaction and image quality as described above.

There are certainly some limitations to the present study. The protocol used is safe, and well tolerable at 1.5 Tesla and provides a good image quality not significantly different from the normal MR spine protocol. Special sequences as DTI or examinations within 3 Tesla magnet bore convey higher energy and thus are like not safe. Another limitation the current study is the small patient population.

\section{Conclusion:}

Using a specifically adapted protocol with a reduced SAR - as presented in the protocol MR examinations at $1.5 \mathrm{~T}$ filed strength can be considered a safe procedure in patients with SCS. The current view that neurostimulators being labelled "not MRI compatible" are still a general contraindication to MR examinations therefore should be questioned. Evaluation of the SCS function before and after MRI is indispensable and further evaluation of other types of IPG and electrodes will increase safety and feasibility of the procedure.

\section{Case report}

A forty year old patient with a history of five times surgery for foraminal stenosis and spinal canal stenosis of the lumbar segment L2/3 and L3/4 with symptoms of axial low back pain and radiculopathy received stabilization and intercorporal fusion L2-4 as ultimate treatment option. For persisting symptoms he underwent SCS implantation. There was a three year course with distinct improvement of symptoms. The patient then became symptomatic with right-sided L5 radiculopathy with pain, hypaesthesia and motor palsy (M4/5). MRI with special neurostimulator protocol was performed and revealed a newly developed disc protrusion L5/S1 with contact to L5 nerve root. After failure of conservative treatment regarding the motor weakness the 
patient underwent sequesterectomy L5/S1. Three month follow-up showed good outcome with complete regression of radicular symptoms and motor weakness. 
Figure 1: Sagittal (A), axial (B) and coronal (C) T2-weighted MRI of patient after two level stabilization and fusion and implantation of SCS 


\section{Tables}

\begin{tabular}{|l|c|}
\hline Number of patients & 13 \\
\hline Number of MRI examinations & 17 \\
\hline Mean patient age [years] & $56.46 \pm 12.79$ \\
\hline Male / Female patients & $9(69.2 \%) / 4(30.7 \%)$ \\
\hline MRI examination performed & CS: 3/ ThS: $1 /$ LS: 13 \\
\hline Number of spinal surgeries before SCS implantation & $2.67 \pm 1.62$ \\
\hline Origin of symptoms & $11(84.6 \% \%)$ \\
\hline - Degenerative spine disease & $1(7.7 \%)$ \\
\hline - Neuropathic pain due to M. Sudeck (LE) & $1(7.7 \%)$ \\
\hline - Neuropathic pain due to CTS &
\end{tabular}

Table 1: General patients' data (per patient)

\begin{tabular}{|l|c|}
\hline Cases of heating of IPG (0-1) & $2(11.8 \%)$ \\
\hline Cases of heating of electrode (0-1) & $1(5.9 \%)$ \\
\hline Pts. experiencing dysesthesia (0-1) & $5(29.4 \%)$ \\
\hline Pts. experiencing pain (0-1) & $4(23.5 \%)$ \\
\hline Overall level of discomfort (1-10) & $2.00 \pm 2.13$ \\
\hline Overall level of satisfaction (1-5) & $1.13 \pm 0.34$ \\
\hline Pts. with dysfunction of SCS after MRI examination & $0(0.0 \%)$ \\
\hline
\end{tabular}

Table 2: Tolerability of MRI examination (Data per examination)

\begin{tabular}{|l|c|}
\hline MRI findings & \\
\hline - High grade spinal canal stenosis & $4(23.5 \%)$ \\
\hline - Disc herniation & $3(17.6 \%)$ \\
\hline - Severe, single segment degeneration & $5(29.4 \%)$ \\
\hline Therapeutic consequences & \\
\hline - Microsurgical discectomy & $1(7.7 \%)$ \\
\hline - Microsurgical spinal canal decompression & $2(15.4 \%)$ \\
\hline - Stabilization and fusion (T-LIF) & $2(15.4 \%)$ \\
\hline - Infiltration (epidural, facet joints) & $3(23.1 \%)$ \\
\hline
\end{tabular}

Table 3: MRI findings and therapeutic consequences

\begin{tabular}{|l|l|l|l|l|l|l|}
\hline & Flip angle & RFPulse & Gradient mode & Turbo factor & TR & SAR \\
\hline Localizer & $20 \mathrm{deg}$ & Low SAR & Whisper & $/$ & 7,3 & $1 \%$ \\
\hline T2_tse_sag & $120 \mathrm{deg}$ & Low SAR & Whisper & 12 & 4120 & $14 \%$ \\
\hline T2_tse_cor & $120 \mathrm{deg}$ & Low SAR & Whisper & 12 & 4040 & $15 \%$ \\
\hline T2_tse_tra & $120 \mathrm{deg}$ & Low SAR & Whisper & 15 & 3200 & $19 \%$ \\
\hline T1_tse_sag & $150 \mathrm{deg}$ & Low SAR & Whisper & 3 & 677 & $37 \%$ \\
\hline
\end{tabular}

Table 5 - Parameters of imaging studies 


\begin{tabular}{|c|c|c|c|c|c|c|c|c|c|}
\hline No & A & S & Type of SCS & $\begin{array}{c}\text { Site of } \\
\text { electrode }\end{array}$ & MRI & MRI findings & $\begin{array}{l}\text { Overall } \\
\text { discomfort }\end{array}$ & $\begin{array}{l}\text { Overall } \\
\text { satisfaction }\end{array}$ & $\begin{array}{l}\text { Therapeutic } \\
\text { consequences }\end{array}$ \\
\hline 1 & 50 & $\mathrm{~F}$ & Prime Advanced & Th9/10 & LS & Facet joint degeneration L5/S1 & 0 & 1 & Infiltration \\
\hline 2 & 44 & $\mathrm{M}$ & Synergy & Th9/10 & LS & Spinal canal stenosis L5/S1 & 3 & 1 & Lu. decompression \\
\hline 3 & 40 & $\mathrm{M}$ & Prime Advanced & Th9/10 & LS & Herniated disc L5/S1 & 4 & 2 & Lumbar discectomy \\
\hline 4 & 45 & $\mathrm{~F}$ & Prime Advanced & Th9/10 & LS & Segment degeneration L5/S1 & 5 & 2 & T-LIF \\
\hline 5 & 48 & $\mathrm{~F}$ & Restore Ultra & Th9/10 & LS & Adjacent segment degeneration & 0 & 1 & Infiltration \\
\hline 6 & 45 & $\mathrm{~F}$ & Itrel 3 & Th9/10 & LS;LS & No new findings & $0 ; 4$ & $1 ; 1$ & none \\
\hline 7 & 75 & $\mathrm{~F}$ & Synergy & Th9/10 & LS & No new findings & 0 & 1 & none \\
\hline 8 & 80 & $\mathrm{M}$ & Prime Advanced & Th9/10 & LS & No new findings & 0 & 1 & none \\
\hline 9 & 63 & $\mathrm{~F}$ & Restore Ultra & Th10/11 & CS;LS & Foraminal stenosis C5, L5 & $1 ; 4$ & $1 ; 1$ & Infiltration \\
\hline 10 & 66 & $\mathrm{M}$ & Restore Ultra & Th10/11 & CS;ThS;LS & Spinal canal stenosis & $0 ; 0 ; 0$ & $1 ; 1 ; 1$ & Lu. decompression \\
\hline 11 & 65 & $\mathrm{~F}$ & Restore Ultra & Th10/11 & LS & No new findings & 6 & 1 & T-LIF \\
\hline 12 & 46 & $\mathrm{~F}$ & Restore Ultra & Th9/10 & LS & Herniated disc L4/5 & 3 & 1 & none \\
\hline 13 & 67 & $\mathrm{~F}$ & Itrel 3 & Th1/2 & CS & Myelopathy & 0 & 1 & none \\
\hline
\end{tabular}

Table 4: Patients overview 


\section{Figure and table legends}

Table 1: General patients' data

Table 2: Tolerability of MRI examination

Table 3: MRI findings and therapeutic consequences

Table 4: Patients overview

Figure 1: Sagittal (A), axial (B) and coronal (C) T2-weighted MRI of patient after two level stabilization and fusion and implantation of SCS

\section{References}

1. Amann W, Berg P, Gersbach P, Gamain J, Raphael JH, Ubbink DT (2003) Spinal cord stimulation in the treatment of non-reconstructable stable critical leg ischaemia: results of the European Peripheral Vascular Disease Outcome Study (SCS-EPOS). Eur J Vasc Endovasc Surg 26:280-286

2. Barolat $G$ (2000) Spinal cord stimulation for chronic pain management. Arch Med Res 31:258-262

3. Claeys LG, Horsch S (1996) Transcutaneous oxygen pressure as predictive parameter for ulcer healing in endstage vascular patients treated with spinal cord stimulation. Int Angiol 15:344-349

4. Cook AW, Oygar A, Baggenstos P, Pacheco S, Kleriga E (1976) Vascular disease of extremities. Electric stimulation of spinal cord and posterior roots. $\mathrm{N} \mathrm{Y}$ State J Med 76:366-368

5. De Andres J, Valia JC, Cerda-Olmedo G, Quiroz C, Villanueva V, MartinezSanjuan V, de Leon-Casasola O (2007) Magnetic resonance imaging in patients with spinal neurostimulation systems. Anesthesiology 106:779-786

6. De Andres J, Villanueva V, Palmisani S, Cerda-Olmedo G, Lopez-Alarcon MD, Monsalve V, Minguez A, Martinez-Sanjuan V (2011) The safety of magnetic resonance imaging in patients with programmable implanted intrathecal drug delivery systems: a 3-year prospective study. Anesth Analg 112:1124-1129

7. Jeon YH Spinal cord stimulation in pain management: a review. Korean J Pain 25:143-150

8. Jivegard LE, Augustinsson LE, Holm J, Risberg B, Ortenwall P (1995) Effects of spinal cord stimulation (SCS) in patients with inoperable severe lower limb ischaemia: a prospective randomised controlled study. Eur J Vasc Endovasc Surg 9:421-425

9. Kainz W, Neubauer G, Uberbacher R, Alesch F, Chan DD (2002) Temperature measurement on neurological pulse generators during MR scans. Biomed Eng Online 1:2

10. Kemler MA, De Vet HC, Barendse GA, Van Den Wildenberg FA, Van Kleef M (2004) The effect of spinal cord stimulation in patients with chronic reflex sympathetic dystrophy: two years' follow-up of the randomized controlled trial. Ann Neurol 55:1318 
11. Klomp HM, Spincemaille GH, Steyerberg EW, Habbema JD, van Urk H (1999) Spinal-cord stimulation in critical limb ischaemia: a randomised trial. ESES Study Group. Lancet 353:1040-1044

12. Liem LA, van Dongen VC (1997) Magnetic resonance imaging and spinal cord stimulation systems. Pain 70:95-97

13. Medical SJ Safety Guidelines. http://sjmneuropro.com.

14. Medtronic (2011) MRI Guidelines.

http://professional.medtronic.com/pt/neuro/scs/ind/mri-

guidelines/index.htm?cmpid=URL Neuro HCP scsmri -\#.UKDVn4VyVTY.

15. Melzack R, Wall PD (1965) Pain mechanisms: a new theory. Science 150:971979

16. Moens M, Droogmans S, Spapen H, De Smedt A, Brouns R, Van Schuerbeek P, Luypaert R, Poelaert J, Nuttin B Feasibility of cerebral magnetic resonance imaging in patients with externalised spinal cord stimulator. Clin Neurol Neurosurg 114:135-141

17. North RB, Kidd DH, Farrokhi F, Piantadosi SA (2005) Spinal cord stimulation versus repeated lumbosacral spine surgery for chronic pain: a randomized, controlled trial. Neurosurgery 56:98-106; discussion 106-107

18. Rezai AR, Finelli D, Nyenhuis JA, Hrdlicka G, Tkach J, Sharan A, Rugieri P, Stypulkowski PH, Shellock FG (2002) Neurostimulation systems for deep brain stimulation: in vitro evaluation of magnetic resonance imaging-related heating at 1.5 tesla. J Magn Reson Imaging 15:241-250

19. Roguin A, Zviman MM, Meininger GR, Rodrigues ER, Dickfeld TM, Bluemke DA, Lardo A, Berger RD, Calkins H, Halperin HR (2004) Modern pacemaker and implantable cardioverter/defibrillator systems can be magnetic resonance imaging safe: in vitro and in vivo assessment of safety and function at $1.5 \mathrm{~T}$. Circulation $110: 475-482$

20. Shealy CN, Mortimer JT, Reswick JB (1967) Electrical inhibition of pain by stimulation of the dorsal columns: preliminary clinical report. Anesth Analg 46:489491

21. Shellock FG (2002) Magnetic resonance safety update 2002: implants and devices. J Magn Reson Imaging 16:485-496

22. Taylor RS, Van Buyten JP, Buchser E (2005) Spinal cord stimulation for chronic back and leg pain and failed back surgery syndrome: a systematic review and analysis of prognostic factors. Spine (Phila Pa 1976) 30:152-160

23. Tronnier VM, Staubert A, Hahnel S, Sarem-Aslani A (1999) Magnetic resonance imaging with implanted neurostimulators: an in vitro and in vivo study. Neurosurgery 44:118-125; discussion 125-116

24. US Food and Drug Administration FDA CfDaRH (2005) FDA Public Health Notification: MRI-Caused Injuries in Patients with Implanted Neurological Stimulators. http://www.fda.gov/MedicalDevices/Safety/AlertsandNotices/PublicHealthNotifications lucm062125.htm.

25. US Food and Drug Administration FDA CfDaRH (1997) A Primer on Medical Device Interactions with Magnetic Resonance Imaging Systems. FDA Public Health Notification www.fda.gov 